\title{
Phase-resolved x-ray ferromagnetic resonance measurements in fluorescence yield
}

\author{
M. K. Marcham, ${ }^{1, a)}$ P. S. Keatley, ${ }^{1}$ A. Neudert, ${ }^{2}$ R. J. Hicken, ${ }^{1}$ S. A. Cavill, ${ }^{3}$ L. R. Shelford,${ }^{3}$ \\ G. van der Laan, ${ }^{3}$ N. D. Telling, ${ }^{4}$ J. R. Childress, ${ }^{5}$ J. A. Katine, ${ }^{5}$ P. Shafer, ${ }^{6}$ and E. Arenholz ${ }^{6}$ \\ ${ }^{1}$ School of Physics, University of Exeter, Stocker Road, Exeter, Devon EX4 4QL, United Kingdom \\ ${ }^{2}$ Forschungszentrum Dresden-Rossendorf e. V., Institute of Ion Beam Physics and Materials Research, \\ P. O. Box 5101 09, 01313 Dresden, Germany \\ ${ }^{3}$ Diamond Light Source, Harwell Science and Innovation Campus, Didcot, Oxfordshire OX11 ODE, \\ United Kingdom \\ ${ }^{4}$ Keele University, Institute for Science and Technology in Medicine, Guy Hilton Research Centre, \\ Thornburrow Drive, Hartshill, Stoke-on-Trent ST4 7QB, United Kingdom \\ ${ }^{5}$ Hitachi Global Storage Technologies, 3403 Yerba Buena Road, San Jose, California 95135, USA \\ ${ }^{6}$ Advanced Light Source, Lawrence Berkeley National Laboratory, Berkeley, California 94720, USA
}

\begin{abstract}
Phase-resolved x-ray ferromagnetic resonance (XFMR) has been measured in fluorescence yield, extending the application of XFMR to opaque samples on opaque substrates. Magnetization dynamics were excited in a $\mathrm{Co}_{50} \mathrm{Fe}_{50}(0.7) / \mathrm{Ni}_{90} \mathrm{Fe}_{10}(5)$ bilayer by means of a continuous wave microwave excitation, while x-ray magnetic circular dichroism (XMCD) spectra were measured stroboscopically at different points in the precession cycle. By tuning the X-ray energy to the $L_{3}$ edges of $\mathrm{Ni}$ and $\mathrm{Fe}$, the dependence of the real and imaginary components of the element specific magnetic susceptibility on the strength of an externally applied static bias field was determined. First results from measurements on a $\mathrm{Co}_{50} \mathrm{Fe}_{50}(0.7) / \mathrm{Ni}_{90} \mathrm{Fe}_{10}(5) / \mathrm{Dy}(1)$ sample confirm that enhanced damping results from the addition of the Dy cap.
\end{abstract}

Precessional dynamics are exploited in the operation of high frequency magnetic devices such as magnetic disk drives, nonreciprocal microwave devices, and spin transfer oscillators. The trajectory of precession and its damping are of crucial importance, and x-ray magnetic circular dichroism (XMCD) has the potential to provide new insight. The chemical specificity of XMCD means that it is possible to isolate the precession in different regions of the sample. By measuring the relative amplitude and phase of oscillation at each chemical species and at distinct sites in the crystal structure, one may in principle determine whether the relaxation takes place preferentially at a specific location within the structure.

X-ray ferromagnetic resonance (XFMR) combines $\mathrm{XMCD}$ and ferromagnetic resonance (FMR) phenomena in a technique capable of measuring the FMR response of an alloy or a multilayer with both chemical and site specificity. Previous XFMR studies can be divided into two distinct categories: phase-resolved measurements ${ }^{1-3}$ and time-averaged measurements. ${ }^{4-6}$ Both types of measurements are generally performed in transmission, although an earlier phaseresolved study was performed in reflection at grazing incidence. $^{7}$ Transmission measurements are usually limited to samples grown on substrates such as $\mathrm{Si}_{3} \mathrm{~N}_{4}$ that are transparent in the soft $\mathrm{x}$-ray regime. In this paper, we present the first phase-resolved XFMR measurements made in fluorescence yield. A $\mathrm{Ta}(5)[\mathrm{Cu}(25) / \mathrm{Ta}(3)]_{3} / \mathrm{Cu}(25) / \mathrm{Ta}(10) / \mathrm{Ru}(5) /$ $\mathrm{Co}_{50} \mathrm{Fe}_{50}(0.7) / \mathrm{Ni}_{90} \mathrm{Fe}_{10}(5) / \mathrm{Ru}(3)$ stack (thicknesses in nanometers) was magnetron sputtered onto an insulating sapphire substrate. A second stack with a Dy(1) layer inserted between the $\mathrm{Ni}_{90} \mathrm{Fe}_{10}(5)$ and the $\mathrm{Ru}(3)$ layers was also deposited. For today's thin sensor layers containing NiFe, the Fe concentration is typically in the range of $8-12 \%$. It is for this reason that we look at $\mathrm{Ni}_{90} \mathrm{Fe}_{10}$ as opposed to the more commonly studied $\mathrm{Ni}_{80} \mathrm{Fe}_{20}$. Elements with $490 \mu \mathrm{m} \times 400$ $\mu \mathrm{m}$ of the ferromagnetic bilayer were formed on the $\mathrm{Cu}$ underlayer by electron-beam lithography and ion beam milling. Photolithography was then used to pattern the exposed $\mathrm{Cu}$ into a coplanar waveguide structure with $50 \Omega$ characteristic impedance. The experimental geometry for the XFMR measurement is shown in Fig. 1. Measurements were performed in the portable octupole magnet system (POMS) on beam line $\mathrm{I} 06$ at the diamond light source (x-ray pulse FWHM $\sim 30 \mathrm{ps}$ ) and in the vector magnet ${ }^{8}$ on beam line 4.0.2 at the advanced light source. Both chambers have an integrated octupole magnet that allows a static magnetic field to be applied in any direction. An in-plane RF magnetic field, of frequency $4 \mathrm{GHz}$, was used to excite the sample magnetization into a state of steady precession about an externally applied static bias field that saturates the sample magnetization along the symmetry axis of the coplanar waveguide. During precession, there are time dependent magnetization components perpendicular to the static bias field. The precession of the magnetization is highly elliptical, with its major axis in-plane, because of the large demagnetizing field found in the ferromagnetic thin film. Sensitivity to this large in-plane component is assured by setting a large angle of incidence for the x-rays. The fluorescence yield is detected using a soft $\mathrm{x}$-ray photodiode (AXUV20A from 


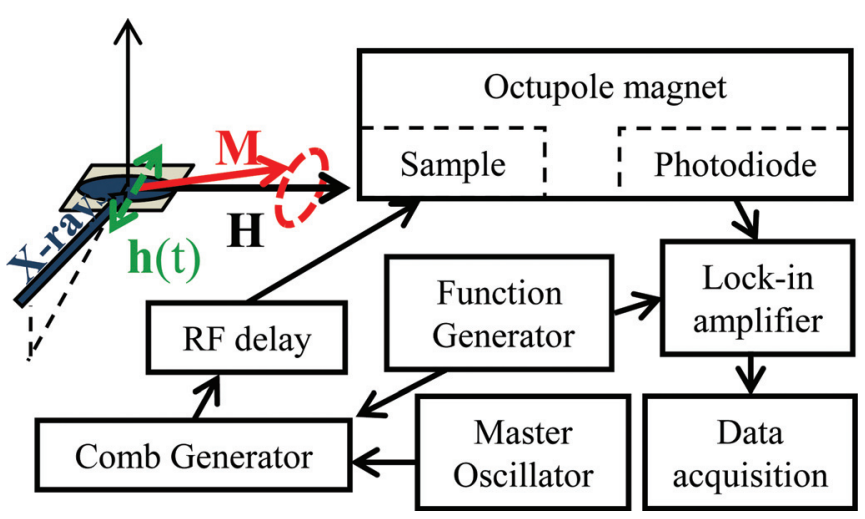

FIG. 1. (Color online) (a) Block diagram of the apparatus and the experimental geometry. Phase resolved measurements are made of the components of the magnetization perpendicular to the static bias field.

International Radiation Detectors) positioned directly above the sample where it subtends a large solid angle. Phaseresolved XFMR measurements require the RF magnetic field and the X-ray pulses to have a fixed phase relation. This is achieved by using a custom comb generator system (Atlantic Microwave Ltd) that takes the $500 \mathrm{MHz}$ synchrotron master oscillator as its input and outputs a specific integer multiple of the master oscillator signal with a fixed phase relation. The phase of the RF excitation can then be adjusted relative to that of the x-ray pulses using a programmable phase shifter with 1 ps resolution (Colby Instrument PDL-100A). The output of the comb generator is amplitude modulated at audio frequencies and the phase-resolved fluorescence signal recovered by passing the output of the photodiode into a lock-in amplifier. To ensure nonzero RF magnetic field amplitude at the sample position for a broad range of excitation frequencies, the center conductor of the coplanar waveguide was shorted at one end so as to provide a current antinode close to the sample position when excited by the comb generator. The central conductor width was $500 \mu \mathrm{m}$, and no heating effects were seen in the presence of the RF current. The magnetic element was positioned $5 \mu \mathrm{m}$ away from the edges of the central conductor to avoid unwanted out of plane excitation. Total electron yield measurements could also be made by measuring the drain current because the RF cabling is electrically isolated from the vacuum chamber.
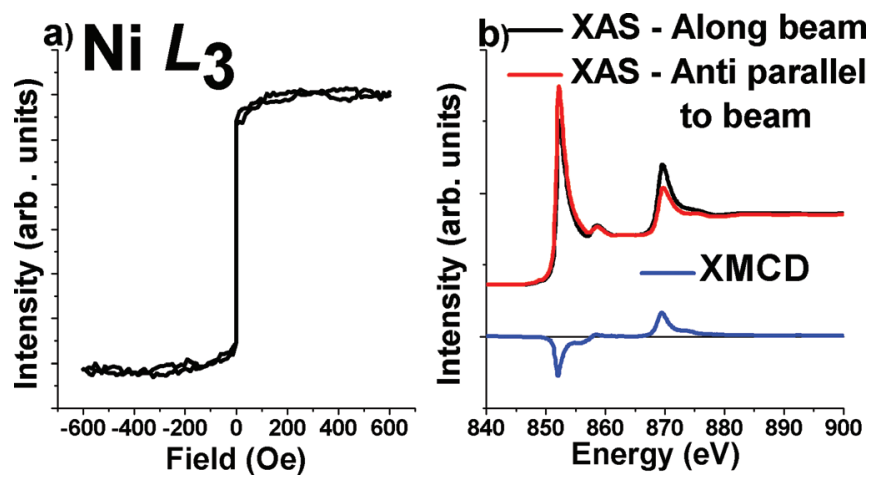

FIG. 2. (Color online) (a) Hysteresis loop taken at the Ni $L_{3}$ edge by sweeping the bias field along the X-ray beam. (b) Ni XAS and XMCD.

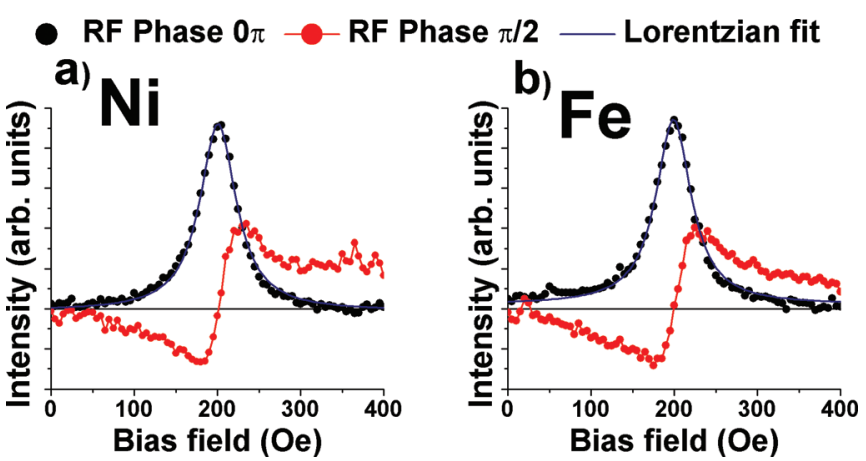

FIG. 3. (Color online) Field sweeps acquired at (a) the $\mathrm{Ni} \mathrm{L}_{3}$ and (b) $\mathrm{Fe} \mathrm{L}_{3}$ edges yield the real and imaginary components of the magnetic susceptibility. Comparison of (a) and (b) confirm that the $\mathrm{Ni}$ and $\mathrm{Fe}$ precess in phase because of the strong exchange coupling.

Tuning the x-ray energy to the $L_{2,3}$ absorption edges leads to the excitation of electrons from the $2 p$ core level to the unoccupied $3 d$ states. There are two main decay channels of the hole created in the $2 p$ core level, namely emission of photons (fluorescence yield) and secondary electrons (electron yield). The latter has a higher probability for the transition metal $L$ edges $^{9}$ but has the undesirable property that the emitted electrons are very sensitive to both RF and static magnetic fields. To ensure that there is no electron yield contribution to the measured signals, a $200 \mathrm{~nm}$ thick aluminum foil (Lebow Co) was placed over the active area of the photodiode. Hysteresis loops are obtained by sweeping the bias

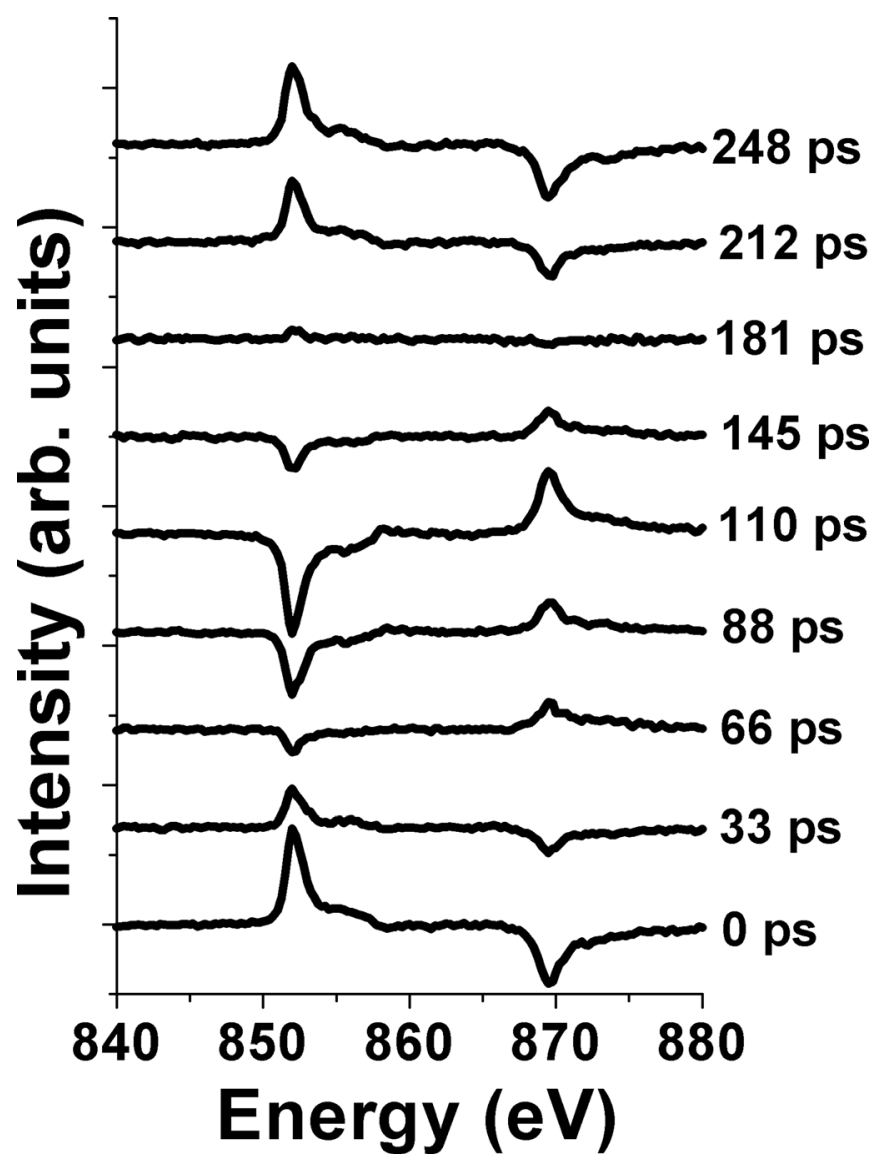

FIG. 4. Phase-resolved Ni XMCD spectra acquired with fixed circular X-ray polarization through one cycle of the $4 \mathrm{GHz}$ excitation. 


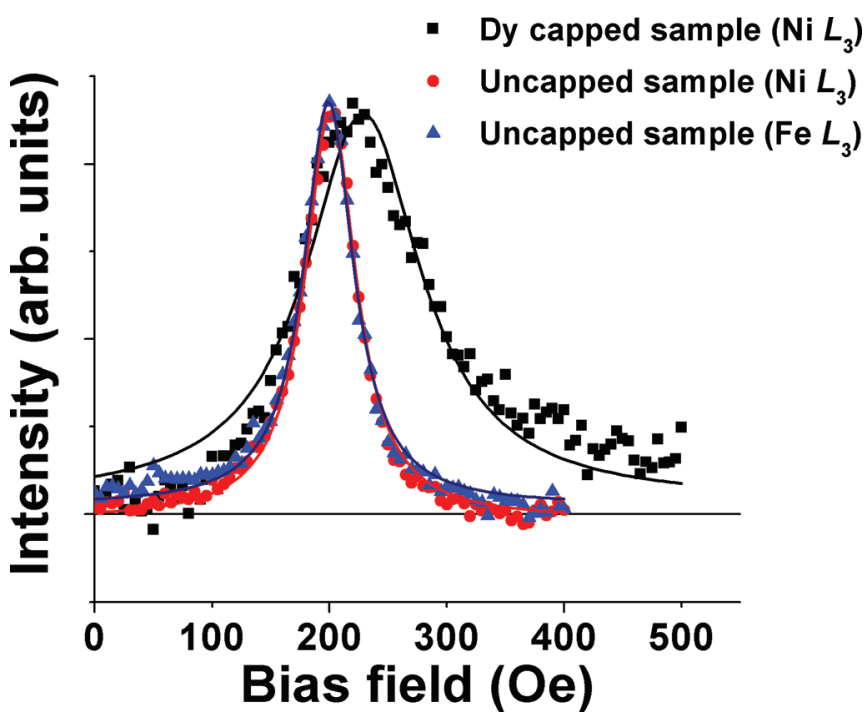

FIG. 5. (Color online) Comparison of measurements performed on capped and uncapped samples confirm the enhanced damping caused by the Dy cap. Lines are Lorentzian fits to the experimental data (symbols).

field with the X-ray energy set to that of the $L$ edge. The $\mathrm{X}$-ray absorption spectroscopy (XAS) and static XMCD signals are obtained by saturating the sample magnetization parallel and antiparallel to the x-ray beam. Typical examples obtained from Ni are shown in Fig. 2.

Phase-resolved measurements are made either by fixing the x-ray energy and sweeping the bias field (field sweeps) or by fixing the bias field and sweeping the x-ray energy (phase-resolved XMCD spectra). Field sweeps performed at the $\mathrm{Ni}$ and $\mathrm{Fe} L_{3}$ edges are shown in Fig. 3. The static bias field is applied along the symmetry axis of the coplanar waveguide, and the scan reveals the bias field at which resonance takes place for a given RF excitation frequency. Measurements are made with the phase between the RF waveform and the $\mathrm{x}$-ray pulses set at two values $\pi / 2 \mathrm{rad}$ apart. This yields signals with lineshapes characteristic of the real and imaginary components of the transverse magnetic susceptibility. Comparison with field sweeps taken at the Fe $L_{3}$ edge confirms that the $\mathrm{Ni}$ and $\mathrm{Fe}$ precess in phase within experimental error, as expected because of the strong exchange coupling between the $\mathrm{Ni}$ and $\mathrm{Fe}$. The resonant bias field is extracted by Lorentzian fitting.

Phase-resolved XMCD spectra are acquired at the resonance field with a fixed circular X-ray polarization. Because the XMCD signal is proportional to the projection of the $\mathrm{X}$-ray wave vector onto the magnetization vector, the phaseresolved XMCD spectra shown in Fig. 4 change sign as the phase of the microwaves, and hence, the precession is shifted by $\pi$ radians.

Recently, it has been shown that capping spin transfer torque oscillators with rare earth materials can lead to increased damping that reduces spin transfer torque noise. ${ }^{10}$
Capping is preferred to doping of the free layer with a rare earth element because the latter could adversely affect the magneto resistance. The initial results shown in Fig. 5 show a clear broadening of the resonance in a Dy capped sample relative to an uncapped sample, confirming the enhanced damping caused by the Dy capping layer. A detailed study combining phase-resolved XFMR measurements in fluorescence yield with Time Resolved Kerr Microscopy (TRKM) and Vector Network Analyzer Ferromagnetic Resonance (VNA-FMR) will be published elsewhere.

At room temperature, bulk Dy is paramagnetic. One might expect some intermixing of the Dy at the interface and possibly a magnetically polarized layer, with the rest of the Dy cap being paramagnetic. Partial ordering of the magnetization of the Dy cap was confirmed by a small measured dichroism ( $0.1 \%$ asymmetry) at the Dy $M_{5}$ edge (not shown). The significant enhancement of the damping can be attributed to the large spin-orbit coupling of the Dy that provides an effective channel for the transfer of angular momentum to the lattice.

We have demonstrated that high quality phase-resolved XFMR measurements may be performed in fluorescence yield XMCD spectra that have been obtained over a complete cycle of $4 \mathrm{GHz}$ RF excitation, and field sweeps performed at the $L_{3}$ edges of $\mathrm{Fe}$ and $\mathrm{Ni}$ yield the real and imaginary parts of the transverse magnetic susceptibility. Comparison of samples with and without a Dy capping layer confirms the presence of an enhanced damping in the former structure. Fluorescence yield measurements may be performed on opaque samples deposited on opaque substrates, widening the application of XFMR measurements.

The ALS is supported by the U.S. Department of Energy under Contract No. DE-AC02-05CH11231The authors gratefully acknowledge the financial support of EPSRC Grant EP/ F021755/1. This work was carried out on beam line I06 at the diamond light source and on beam line 4.0.2 at the advanced light source.

${ }^{1}$ Y. Guan, W. E. Bailey, E. Vescovo, C.-C. Kao, and D. A. Arena, J. Magn. Magn. Mater. 312, 374 (2007).

${ }^{2}$ D. A. Arena, E. Vescovo, C.-C. Kao, Y. Guan, and W. E. Bailey, Phys. Rev. B 74, 064409 (2006).

${ }^{3}$ T. Martin, G. Woltersdorf, C. Stamm, H. A. Dür, R. Maththeis, C. H. Back, and G. Bayreuther, J. Appl. Phys. 105, 07D310 (2009).

${ }^{4}$ J. Goulon, A. Rogalev, F. Wilhelm, N. Jaouen, C. Goulon-Ginet, G. Goujon, J. Ben Youssef, and M. V. Indendom, JETP Lett. 82, 696 (2005).

${ }^{5}$ G. Boero, S. Rusponi, P. Bencok, R. S. Popovic, H. Brune, and P. Gambardella, Appl. Phys. Lett. 87, 152503 (2005).

${ }^{6}$ G. Boero, S. Mouaziz, S. Rusponi, P. Bencock, F. Nolting, S. Stepanow, and P. Gambardella, New J. Phys. 10, 013011 (2008).

${ }^{7}$ W. E. Bailey, L. Cheng, D. J. Keavney, C.-C. Kao, E. Vescovo, and D. A. Arena, Phys. Rev. B 70, 172403 (2004).

${ }^{8}$ E. Arenholz and S. O. Prestemon, Rev. Sci. Instrum. 76, 083908 (2005).

${ }^{9} \mathrm{~J}$. Stohr and H. C. Siegmann, Magnetism from Fundamentals to Nanoscale Dynamics (Springer, Berlin, 2006).

${ }^{10}$ S. Maat, N. Smith, M. J. Carey, and J. R. Childress, Appl. Phys. Lett. 93, 103506 (2008). 
This document was prepared as an account of work sponsored by the United States Government. While this document is believed to contain correct information, neither the United States Government nor any agency thereof, nor the Regents of the University of California, nor any of their employees, makes any warranty, express or implied, or assumes any legal responsibility for the accuracy, completeness, or usefulness of any information, apparatus, product, or process disclosed, or represents that its use would not infringe privately owned rights. Reference herein to any specific commercial product, process, or service by its trade name, trademark, manufacturer, or otherwise, does not necessarily constitute or imply its endorsement, recommendation, or favoring by the United States Government or any agency thereof, or the Regents of the University of California. The views and opinions of authors expressed herein do not necessarily state or reflect those of the United States Government or any agency thereof or the Regents of the University of California. 